\title{
Berberine Interferes With the Abnormal Glomerular Mesangial Cell C Ycle Re-Distribution to Alleviate Diabetic Nephropathy via PI3K/AKT/AS160/GLUT4 Signaling Pathway
}

\section{Ximei Guan}

The First Affiliated Hospital of USTC: Anhui Provincial Hospital

Weijian Ni ( $\nabla$ niweijian@ustc.edu.cn )

Anhui Medical University https://orcid.org/0000-0002-9844-0578

Jing Zeng

Anhui Provincial Hospital

Hong Zhou

Anhui Provincial Cancer Hospital

Linqin Tang

The First Affiliated Hospital of USTC: Anhui Provincial Hospital

\section{Research article}

Keywords: Berberine, diabetic nephropathy, glomerular mesangial cells, PI3K/AKT, GLUT4, cell cycle redistribution

Posted Date: February 11th, 2021

DOI: https://doi.org/10.21203/rs.3.rs-181818/v1

License: (c) (i) This work is licensed under a Creative Commons Attribution 4.0 International License.

Read Full License 


\section{Abstract}

Background: Berberine plays a critical role of the glomerular mesangial cells (GMCs) abnormal proliferation during diabetic nephropathy (DN). This study aims to explore the intervention effect of BBR on DN mice and investigate the potential mechanism targeting abnormal GMCs proliferation.

Methods: Streptozotocin-induced mice were used to determine the effect of BBR on the renal injury. In vitro, GMCs are cultured in high glucose $(30 \mathrm{mmol} / \mathrm{L})$. EdU and MTT assay are used for screening the optimum BBR concentration and intervention time. Flow cytometry is applied to analyze the cell cycle redistribution. Western blot and RT-qPCR are devoted to studying the relative expression of molecules in PI3K/AKT/AS160/GLUT4 signaling pathway. Additionally, 2-NBDG assay is selected for measuring the glucose uptake of GMCs.

Results: HE and PAS staining revealed that the notable mesangial matrix expansion, glomerular hypertrophy and glycogen deposition in diabetic kidney can be alleviated after BBR treatment. Moreover, BBR significantly reduced the positive expression of GLUT4 in tubulointerstitium and glomerular region. EdU shows that high glucose induces the abnormal proliferation of GMCs, which becomes more apparently as time goes on $(20 \mathrm{~h} \rightarrow 28 \mathrm{~h})$. Meantime, BBR $(60$ and $90 \mu \mathrm{mol} / \mathrm{L})$ can not only increase the proportion of $\mathrm{G} 1$ phase, but also reduce the proportion of $S$ phase. After $24 \mathrm{~h}$, the same sort of phenomenon has cropped up in BBR $(30 \mu \mathrm{mol} / \mathrm{L})$ group. BBR $(60 \mu \mathrm{mol} / \mathrm{L}))$ significantly degraded the levels of PI3K-p85, p-AKT, p-AS160, and the membrane-GLUT4, while indistinguishable changes of their total protein expressions. Additionally, BBR prominently reduced the glucose uptake and retard the cell cycle of GMCs to stay at G1 phase.

Conclusions: The rearrangement effect of BBR on cell cycle is related with PI3K/AKT signaling pathway and GLUT4 trafficking. The key findings of our study collectively indicate that the treatment of GMCs proliferation can be a novel therapeutic strategy in DN.

\section{Background}

Diabetic nephropathy (DN), one of the most frequent and serious complications of diabetes mellitus, which is considered as a principal cause account for end-stage renal disease (ESRD) (Perkovic V et al.,2019). Clinically, cell destiny shift (such as abnormal glomerular mesangial cells (GMCs) proliferation and podocyte apoptosis), histopathological changes (including glomerular hypertrophy, sclerosis, basement membrane thickening and renal fibrosis) are the main characteristics of DN largely dependent on metabolic disorders (Fu H et al., 2019; Clark AJ et al., 2020). The number of patients with DN is still on the rise worldwide, and this requires the development of new therapeutic strategies. Numerous studies discover that the complex pathogenesis of DN has multiple pathogenic factors, such as hyperglycemia, hyperlipidemia, hemodynamic abnormalities, etc (Qi MY et al., 2020; Perkins BA et al., 2019). But in the end, hyperglycemia is still regarded as the basic and key factor to accelerate the progress of DN (Tziomalos $\mathrm{K}$ et al., 2015). In a number of studies, hyperglycemia can induce oxidative stress, 
mitochondria dysfunction and insulin resistance, which promotes the occurrence of DN (Han Y et al., 2018; Coughlan MT et al., 2016). Besides, hyperglycemia affects blood pressure and then accelerates the progression of DN (Vallon V et al., 2017). Probing further, they notice that hyperglycemia is one of the important factors during the abnormal GMCs proliferation in the diabetic kidney (Qi C et al., 2017). Glucose is the major source of energy for most mammalian cells and is particularly important during the fetal development when the cells are rapidly dividing and differentiating (Wang AJ et al., 2019). However, a high glucose $(\mathrm{HG})$ concentration may be a harmful effect on cell growth. GMCs are an important component of the glomerulus, which can act as the barrier of the glomerulus (Ji TT et al., 2020). Notably, GMCs proliferation has been prominently identified in DN animal models, and appear to have a key role in the induction of mesangial matrix expansion, hypercellularity, and the onset of proteinuria (Sagoo MK et al., 2018). Previously, it has been reported that GMCs proliferation arises in part due to abnormally high cellular glucose uptake in response to a high extracellular glucose concentration by glucose transporters (Kanwar YS et al., 2008). Glucose transporters (GLUTs) are a large cluster of membrane proteins that facilitate the transport of glucose through the cellular plasma membrane (Sagoo MK et al., 2018). According to a number of studies, GLUT4 is the only transporter responsible for facilitating glucose transport into cells in response to insulin and is considered as a vital regulator of entire body glucose homeostasis (Thorens B et al., 2010; Lv Y et al., 2020). The role of GLUT4 in the GMCs abnormalities that occur in response to the $\mathrm{HG}$ environment is less well understood. Thus, the effective control of blood glucose levels is critical to delay the progression of GMCs proliferation and to reduce the risk of DN (Kushwaha K et al., 2020). Although studies have already paid dividends, the molecular and cellular mechanisms underlying the GMCs proliferation are still unknown.

Phosphatidyl inositol 3 kinase/protein kinase B (PI3K/AKT) pathway is reported as one of the most important pathways involved in many cellular processes including proliferation, differentiation, apoptosis, tumor growth and cell cycle progression (Xia X et al., 2020; Molinaro A et al., 2019). In general, PI3K/AKT pathway can be activated by a range of signals, including cancer development, insulin metabolism, growth factors and components of excessive extracellular matrix (ECM), which will lead to the launch of the AKT signaling cascade. After activation, Akt targets several downstream molecules to alter their activities by phosphorylation or complex formation (Zhang $\mathrm{H}$ et al., 2014). Several studies find that AKT is involved in cell proliferation through interaction with a number of proteins involved in the cell cycle (Lu CC et al., 2017). GLUT4 is regulated by the PI3K/AKT signaling pathway, which is activated in diabetes. AKT modulates insulin-regulated glucose metabolism, which facilitates GLUT4 translocation from cellular stores to the plasma membrane. So, the activation of PI3K/AKT signaling is related with DN. Meantime, evidence from other studies suggests that AS160 (AKT substrate of $160 \mathrm{kDa} / \mathrm{Tbcld} 4$ ) acts as the substrate of insulin signal related AKT, which plays an important role in regulating glucose metabolism and improving insulin resistance (Young LH et al., 2000). It has been reported that the AS160 has the ability to bind to GLUT4 vesicles as well as the plasma membrane, although the molecular details of these interactions have not yet been elucidated (Larance $M$ et al., 2005). Based on previous researches, we found that $\mathrm{HG}$ can induce the podocyte apoptosis through regulating PI3K/AKT signaling pathway 
(Fruman DA et al., 2017). Meantime, abnormal GMCs proliferation occurred under HG condition from the result of a preliminary experimental study (Tang D et al., 2018; zhang $J$ et al., 2018).

According to these results, many questions out through our brain: whether there is any relationship between HG-induced abnormal GMCs proliferation and PI3K/AKT signaling pathway alteration? If possible, what is the possible mechanism within?

In recent years, a growing number of studies have discovered the potential value of Chinese traditional medicine in diseases' treatment (Hao P et al., 2017). Berberine (BBR), a famous herb extract generally isolated from rhizoma coptidis rhizomes and cortex phellodendri, has been regarded as a potential and promising drug for preventing diabetes and its complications (Wu Z et al., 2020; Shinjyo N et al., 2020). Several compelling studies have found BBR could not only reduce the level of HG, regulate the abnormal blood lipid, attenuate kidney inflammation, but also improve insulin resistance and enhance insulin activity under the diabetic condition in animal models (Zhang $J$ et al., 2018). In the meantime, BBR could obviously regulate the balance of podocyte injury and autophagy by affecting the PI3K/AKT pathway, which could postpone the development of DN (Zhang $\mathrm{H}$ et al., 2014). However, the effect of BBR on GMCs in DN remains to be further studied. Therefore, in this study, we explore internal relations and molecular mechanisms among the protective effect of BBR, HG-induced abnormal GMCs proliferation, and the PI3K/AKT signaling pathway. Notably, our study will provide significant insights into the mechanisms controlling cellular responses to hyperglycemia that initiate the progression of DN.

\section{Materials And Methods}

\section{Materials}

Streptozotocin (STZ, S0130) was obtained from Sigma Chemical company (Louis, MO, USA). GMCs (147589) were purchased from Shanghai Cell Bank of Chinese Academy of Sciences (Shanghai, China). BBR (181013) was got from BeNa Culture Collection (Beijing, China). MTT (M8180) and 0.4\% Trypan blue solution (ST798) were obtained from Solarbio (Beijing, China). Membrane and Cytosol Protein Extraction Kit (P0033), EdU (5-ethynyl-2-deoxyuridine) (C0071S) and Cell cycle detection kit (BB-4104) were acquired from Beyotime Biotechnology (Shanghai, China). 2-NBDG (N13195) was obtained from Thermo Fisher Scientific (Carlsbad, USA). Rabbit anti-PI3K-p85 (60225-1-lg) was got from Proteintech (60225-1-lg, Wuhan, China), while rabbit anti-AKT (4691S), anti-phospho-AKT (ser473), anti-GAPDH (5174), antiAS160 (2670), anti-phospho-AS160 (8619) antibodies were obtained from Cell Signaling Biotechnology (Boston, MA, USA). Rabbit anti-GLUT4 antibody (48547) was bought from Abcam Biotechnology (Cambridge, Cambridge shire, UK). All other chemicals applied in these experiments were belong to analytical grade and were acquired from commercial sources.

\section{Animals}

C57BL/ 6 male mice were selected as subjects. Mice $(n=50)$ were fed with a standard diet and water ad libitum and adapted to the experimental conditions $\left(20 \pm 2^{\circ} \mathrm{C}\right.$, humidity of $\left.60 \pm 5 \%\right)$ for 7 days. Then, they 
were randomly divided into five groups: normal group ( $n=10, N C), D N$ model group $(n=10, D N)$, DN mice treated with BBR $(n=10,90 \mathrm{mg} / \mathrm{kg})$ group, BBR $(n=10,180 \mathrm{mg} / \mathrm{kg})$ group, and Metformin $(n=10,200$ $\mathrm{mg} / \mathrm{kg}$ ) group. Before the start of the experiment, 10 mice were assigned to the NC group with only normal diet. Other mice were fed with high glucose/ lipid diet for 6 months and received intraperitoneal injections of streptozotocin ( $50 \mathrm{mg} / \mathrm{kg} /$ day) for 5 consecutive days to build type II diabetic model. The development of hyperglycemia (type II diabetes) in mice was confirmed by estimating the fasting blood glucose (FBG) level ( $\geq 16.7 \mathrm{mmol} / \mathrm{L})$ after $72 \mathrm{~h}$. Control mice given vehicle alone showed a normal range of 5-6 mmol/L. Mice in the NC and DN groups were given an equal volume of vehicle (CMC-Na), while the BBR treatment groups were received BBR dissolved in $0.5 \%$ carboxymethyl cellulose at doses of 90 $\mathrm{mg} / \mathrm{kg}, 180 \mathrm{mg} / \mathrm{kg}$ per day for 10 weeks intragastrically. After 10 weeks, kidney samples were rapidly and frozen in liquid nitrogen or fixed in $10 \%$ formaldehyde buffer. The animal experiment has been ethically acceptable, and where relevant conforms to national guidelines for animal usage in research.

\section{Histopathology and immunohistochemistry}

After dissection of the animals, one entire kidney was placed in $10 \%$ neutral formalin solution. Sections (3-5 $\mu \mathrm{m})$ were taken, processed and stained with HE staining to detect the structure of kidney and periodic acid-Schiff (PAS) reagent to detect glycogen. For immunohistochemistry, renal sections were dewaxed in xylene and dehydrated with an ethanol gradient. After antigen retrieval by microwaving in $0.1 \mathrm{M}$ citric saline at $95^{\circ} \mathrm{C}$ for $3 \mathrm{~min}$, sections were incubated with peroxide blocking reagent for $10 \mathrm{~min}$, rinsed with phosphate buffer and again incubated with power block solution for 10 min. Non-specific binding was minimized by leaving the sections in 3\% BSA in phosphate buffered saline for $30 \mathrm{~min}$. Sections were incubated overnight with a 1:200 dilution of GLUT4 antibody. Finally, they were rinsed well with phosphate buffer and incubated in a supersensitive polymer-horseradish peroxidase immunohistochemistry detection system. Then, the sections were washed with buffer and incubated with diaminobenzidine substrate solution for $5 \mathrm{~min}$, and subsequently were counterstained with hematoxylin and detected using Leica Biosystems.

\section{Transmission electron microscopy (TEM)}

Kidney tissues were fixed in $2.5 \%$ glutaraldehyde and then osmic acid for $12 \mathrm{~h}$. The sections were dehydrated in alcohol (from 50\% to $90 \%$ ) and araldite sequentially. Finally, the tissues sections were embedded in pure Araldite. Ultrathin sections $(70 \mathrm{~nm})$ were cut with ultra-microtome (UC-7, Leica) and mounted on a copper grid (200 mesh). The sections were stained with uranyl acetate and lead citrate. The transmission electron microscope (JEM1400, Japan) was used for viewing and morada G3 was used for photographing.

\section{Cell culture}

GMCs were cultivated in Dulbecco's modified Eagle's medium (DMEM) supplemented with $10 \%$ fetal bovine serum (FBS), 100 units $/ \mathrm{mL}$ penicillin and $100 \mu \mathrm{g} / \mathrm{mL}$ streptomycin at $37^{\circ} \mathrm{C}$ in a humidified atmosphere containing $5 \% \mathrm{CO}_{2}$. Serial passaging was performed when the cells reached $80 \%$ confluence. 


\section{MTT assay and cell survival}

The viability of GMCs was measured by 3-(4, 5-Dimethylthiazol-2-yl)-2, 5-diphenyltetrazolium bromide (MTT). Briefly, cells $\left(1.0 \times 10^{4}\right.$ cells per well) were seeded into 96 -well plates and incubated at $37^{\circ} \mathrm{C}$ overnight. After that, cells were pre-treated with serum-free medium for $12 \mathrm{~h}$ and subsequently cultured with additional D-glucose (30 mmol/L) or serum-free normal glucose medium for different hours $(12,24$, $36,48 \mathrm{~h}$ ). The absorbance was measured by a microplate reader (SpectraMax i3x, Molecular Devices, Shanghai, China) at $490 \mathrm{~nm}$ according to the operations manual. Cell viability was expressed as the percentage of viable cells relative to that of the non-treated control cells. All results were repeated for three times.

\section{Trypan blue}

GMCs were cultured under different concertations of high glucose condition and treated with BBR at different times. After treatment, the cell suspension mixed with $0.4 \%$ trypan blue solution at volume ratio 9:1. Then, countess ${ }^{\circledR}$ cell counting chamber slides were inserted into the instrument. The dead cells were stained blue, and the live cells were colorless and transparent.

\section{Flow cytometry analysis the cell cycle}

GMCs were washed twice with cold PBS and fixed in cold $70 \%$ ethanol overnight at $4^{\circ} \mathrm{C}$. Fixed cells were washed with cool PBS and stained using the cell cycle assay kit. The experiment was repeated three times. Finally, the samples were analyzed on a Beckman flow cytometer, and data were collected for 10,000 single cell events. The percentage of cells in the G1, S and G2 phases of the cell cycle was determined by CytExpert software. Finally, data are presented as histograms.

\section{Fluorescent EdU analysis}

GMCs with a density of $1 \times 10^{4}$ cells/well were cultured in six-well plate for $24 \mathrm{~h}$, then EDU assay was performed using fluorescence microscopy (DM2000, Leica, Germany) after different treatment in different groups. Briefly, the cells were fixed with $4 \%$ paraformaldehyde (PFA) for $15 \mathrm{~min}$ at room temperature, and then permeabilized by $0.3 \%$ Triton-X100 for $15 \mathrm{~min}$, following the click reaction solution was added and incubated at room temperature for $30 \mathrm{~min}$ in the dark. Following this, samples were washed with PBS. Then, cells were stained with Hoechst 33342 for 10 min at room temperature. Finally, the images were obtained with a microscope and were analyzed with Image-Pro plus. The EDU incorporation rate was calculated as the ratio of EDU positive cells (green cells) to total Hoechst positive cells (blue cells).

\section{Western blotting analysis}

After treated with indicated drugs, the cells were washed with $1 \mathrm{ml}$ ice-cold PBS. To examine the translocation of GLUT4, we extracted and isolated the cytoplasmic and membrane protein were performed according to the Membrane and Cytosol Protein Extraction Kit\Beyotime, Jiangsu, China $\$.Cell and tissue total proteins were extracted or isolated according to the manufacturer protocols. Proteins 
were separated by SDS-PAGE and transferred to a PVDF membrane (Millipore Corporation, Bedford, MA). The PVDF membranes were blocked with $5 \%$ nonfat milk powder for $2 \mathrm{~h}$ at room temperature and then incubated with the appropriate primary and secondary antibodies. Anti-PI3K-p85 antibody (1: 1000), antiAKT antibody (1: 1000), anti-p-AKT antibody (1: 1000)『anti-AS160 antibody (1: 1000), anti-p-AS160 antibody (1: 1000) and anti-GLUT4 antibody (1: 1000) were used as primary antibodies. All of the immunoblots were detected with HRP western blotting detection reagents (Millipore Corporation. Equal loading was confirmed using an anti-GADPH antibody (1: 1000).

\section{RT-qPCR analysis}

Total RNA was extracted from GMCs with TRIzol ${ }^{\circledR}$ reagent (Invitrogen; Thermo Fisher Scientific, Inc). RNA concentrations were measured using analytikjena (Germany, Jena). RNA samples with an A260/A280 ratio of 1.8-2.0 were used for next steps. To detect the relative mRNA level, a Transcription First Strand cDNA Synthesis Kit (Vazyme Biotech, China) was used to perform the reverse transcription. Reaction conditions were as follows: $50^{\circ} \mathrm{C}$ for $15 \mathrm{~min}, 85^{\circ} \mathrm{C}$ for 5 seconds. A PCR reaction system was prepared using SYBR ${ }^{\circledR}$-Green Real-Time PCR Master mix (Thermo Fisher Science ABI7500). PCR reaction conditions were as follows: $95^{\circ} \mathrm{C}$ for $10 \mathrm{sec}$, followed by 40 cycles of $10 \mathrm{sec}$ at $95^{\circ} \mathrm{C}$ and $30 \mathrm{sec}$ at $60^{\circ} \mathrm{C}$. The GAPDH was served as an internal control to normalize the relative expression of PI3K, AKT, GLUT4 and AS160. All data were quantified using the $2^{-\Delta \Delta C t}$ method and run in triplicate for each sample. Primer sequences used in RT-qPCR are listed in Table 2.

\section{2-NBDG assay for glucose uptake}

2-NBDG (2-(N-(7-Nitrobenz-2-oxa-1,3-diazol-4-yl) Amino)-2-Deoxyglucose), a fluorescent glucose analog that has been used to monitor glucose uptake in live cells, as an indicator of cell viability. The GMCs were seeded at a concentration of $1 \times 10^{5} \mathrm{cells} / \mathrm{mL} /$ well in duplicate in a six wells plate and incubated overnight at $37^{\circ} \mathrm{C}$. The cells were washed twice with cold KBR after high glucose treatment, then were cultured with 2-NBDG for 30 min at $37^{\circ} \mathrm{C}$. The last, cells were washed twice with cold KBR buffer to halt glucose uptake, and resuspend with KBR to fluorescence detection using a flow cytometer at a fluorescence excitation of $488 \mathrm{~nm}$ and emission of $520 \mathrm{~nm}$. The experiment was performed in triplicate.

\section{Statistical analysis}

Data were assessed using SPSS 24.0 (IBM Corporation, Armonk, NY, USA). Significant differences were evaluated using one-way ANOVA using a post hoc Bonferroni correction (GraphPad Prism 5.0; GraphPad Software, La Jolla, CA, USA). A two-sided $p<0.05$ was considered significant. The data are presented as the mean $\pm S D(n \geq 3)$.

\section{Results}

\section{BBR ameliorates renal pathological changes in DN mice model}


The renoprotective effect of BBR was accessed by HE, PAS and TEM. HE staining was conducted to detect the renal pathological changes. As shown in Fig. 1A, the kidney from DN mice revealed obvious widened, the epithelial cells were severely damaged, and the tubular basement membrane was broken and resembled bristles. PAS staining is often used to semi-quantify glycogen (Fig. 1B). In this study, the technique was used to examine the accumulation of mesangial matrix in mice as its components were mainly composed of polysaccharide-associated agents. Further examination of TEM (Fig. 1C), the normal ultrastructure of the glomeruli was observed in the kidney tissue of the NC group, such as the renal glomerular structure integrity was completed, basement membrane thickness was found no obvious hyperplasia. Podocyte was not seen as obvious abnormity, and the morphological structure was wellbalanced. Compared with the NC group, the glomerular basement membrane was thickened obviously, podocytes foot processes fusion, disordered arrangement. Administration of BBR $(90 \mathrm{mg} / \mathrm{kg}), \mathrm{BBR}(180$ $\mathrm{mg} / \mathrm{kg}$ ) and Metformin $(200 \mathrm{mg} / \mathrm{kg})$ could normalize the above-mentioned abnormal alterations of model group to a certain degree.

\section{BBR changes of PI3K/AKT signaling pathway in DN mice}

The relative expression of protein including PI3K, AKT and p-AKT were measured with western blot. As shown in Figure, the expression of PI3K-p85, p-AKT were significantly increased in DN mice compared with normal mice. Data statistically point out that treating different dosages of BBR can remarkedly downregulated the expression of PI3K-p85 and p-AKT. Western blot analysis demonstrated that in the process of DN, PI3K/AKT signaling pathway was motivated. Notably, BBR inhibited the activity of $\mathrm{PI} 3 \mathrm{~K} / \mathrm{AKT}$ signaling (Fig. 2).

\section{BBR inhibits the expression of GLUT4 in the kidney tissue of DN mice}

GLUT4 protein presence was detected in the renal glomerular and tubular interstitial by immunohistochemistry (Fig. 3). The expression of GLUT4 in the DN group significantly increased compared with the NC group. Treatment with BBR (90 and $180 \mathrm{mg} / \mathrm{kg}$ ) ameliorated this effect, the same effect as Metformin $(200 \mathrm{mg} / \mathrm{kg})$ group.

\section{BBR inhibits HG-induced abnormal GMCs proliferation}

GMCs are used to establish models in HG conditions, while those in normal glucose are taken as normal control (NC). Then, MTT assay and trypan blue staining are used to determine the cell proliferation characteristics and screen the appropriate BBR intervention concentration and time. As MTT and trypan blue staining results show, $\mathrm{HG}$ culture can significantly increase the abnormal GMCs proliferation at $24 \mathrm{~h}$ and this situation continues with time $(24 \mathrm{~h} \rightarrow 48 \mathrm{~h})(\mathrm{p}<0.001)$ compared with NC group (Figure 4B), while the above phenomenon is not caused by normal glucose (Figure 4A). Meantime, BBR $(30,60$, and 90 $\mu \mathrm{mol} / \mathrm{L}$ ) can be considered as the appropriate candidate concentration for the first $24 \mathrm{~h}$ of treatment after considering the inhibitory effect (Figure 4B) and cytotoxicity effect as shown in Figure 3A and Table 1. In order to precisely understand the abnormal GMCs proliferation characteristics and decide the intervention time for the next step, we use EdU assay to detect and find that the unapparent and apparent abnormal 
GMCs proliferation occurs at $20 \mathrm{~h}(\mathrm{p}<0.05)$, respectively, and therefore, $12 \mathrm{~h}, 16 \mathrm{~h}, 20 \mathrm{~h}, 24 \mathrm{~h}, 28 \mathrm{~h}$ intervention times are included in the following experiment (Fig. 5).

\section{BBR regulates cell cycle redistribution of GMCs}

In this section, we use flow cytometry to explore the effect of HG and BBR on regulating the cell cycle redistribution of GMCs (Fig. 6A). Results show that HG significantly prevents the GMCs enter into G1 phase $(p<0.05)$ and accelerate the $G 1$ cells enter into $S$ phase $(p<0.001)$, while has no remarkable influence on $\mathrm{G} 2$ phase ( $p>0.05$ ) (Fig. 6B). After treatment at $12 \mathrm{~h}$ and $16 \mathrm{~h}$, almost no cell cycle re-distribution changes in GMCs (p>0.05) (Fig. 6C-D). Then, as shown as Fig. 6E, BBR (60 and $90 \mu \mathrm{mol} / \mathrm{L})$ can not only increase the proportion of G1 phase, but also reduce the proportions of GMCs in S phase at 20h, and this tendency becomes more significant as time goes on $(\mathrm{p}<0.001)$. After $24 \mathrm{~h}$ and $28 \mathrm{~h}$, the same sort of phenomenon has cropped up in the BBR $(30 \mu \mathrm{mol} / \mathrm{L})$ group $(\mathrm{p}<0.001)$, which can be seen in Fig. $6 \mathrm{~F}-\mathrm{G}$. Therefore, we choose BBR $(60 \mu \mathrm{mol} / \mathrm{L})$ as the most appropriate intervention concentration in the next steps after comprehensive consideration.

\section{BBR regulates $\mathrm{PI3K} / \mathrm{AKT} / \mathrm{AS} 160 /$ GLUT4 signaling pathway}

When exploring the effect of BBR of PI3K/AKT/AS160/GLUT4 signaling pathway, BBR (60 $\mu \mathrm{mol} / \mathrm{L})$ and LY294002 are selected as appropriate intervention concentration and PI3K inhibitor, respectively. As shown in Fig. 7A-B, BBR can significantly inhibit the HG-induced elevated protein levels of PI3K-p85, phosphorylated AKT (p-AKT), phosphorylated AS160 (p-AS160), and the membrane-GLUT4, while has little effect on the total protein expressions at 20h. Meantime, BBR also suppresses the mRNA expression of these parameters as shown in QPCR result (Fig. 7C-F). These results indicated BBR can inhibit the signal transduction of PI3K/AKT/AS160/GLUT4 pathway in GMCs cultured under HG condition.

\section{BBR affects GLUT4 mediated glucose uptake, cell cycle re-distribution and abnormal GMCs proliferation}

Based on the above results we learn that BBR could inhibit the signal transduction of $\mathrm{PI3K} / \mathrm{AKT} / \mathrm{AS} 160 / \mathrm{GLUT} 4$ pathway to reduce the level of membrane GLUT4. In Fig. 8A-B, we use 2-NBDG assay to measure the glucose uptake and find that HG significantly increases the glucose uptake of GMCs $(p<0.001)$, while this effect is reversed by BBR $(60 \mu \mathrm{mol} / L)$ and LY294002 treatment $(p<0.001)$. Meantime, BBR $(60 \mu \mathrm{mol} / \mathrm{L})$ markedly regulate cell cycle re-distribution and inhibit the abnormal cell proliferation at $20 \mathrm{~h}$, specifically as follows: BBR not only increase the proportion of $\mathrm{G} 1$ phase, but also arrest the GMCs in $\mathrm{G} 1$ phase enter into $S$ phase (Fig. 8C-D) ( $<<0.001)$, which can notably inhibit the abnormal GMCs proliferation cultured in HG condition (Fig. 8E-F) $(p<0.01)$.

\section{Discussion}

DN is an ordinary complication of diabetes mellitus which is the leading cause of end-stage renal disorder, posting a menacing threat to public health, due to it caused multiple pathological changes (Tervaert TW et al., 2010). Among these alterations, HG-induced proliferation and ECM deposition of the 
mesangial cell have been identified as the prominent pathological features in the progression of DN (Xie R et al., 2017; Tsai YC et al., 2020). It is worth noting that GMCs abnormal proliferation may be present in DN patients before the onset of clinical manifestations (Turgut $F$ et al., 2010). In our prophase research, we find that functional alterations in GMCs in response to high extracellular glucose levels have been involved in the pathophysiology of DN (Ni WJ et al., 2016; Qiu YY et al., 2017). The increased intracellular glucose concertation results in activation of several pathophysiologic mechanisms, including the metabolic polyol pathway, increased synthesis of TGF- $\beta 1$, intracellular production of advanced glycation end products, and the generation of reactive oxygen species (Tsai YC et al., 2020). All of these effects contribute to the increased synthesis of the mesangial matrix, fibrogenesis components such as fibronectin and collagen, leading to mesangial expansion and glomerular sclerosis. However, the detailed mechanism of abnormal GMCs proliferation under such conditions is still unclear, which urges us to explore the details.

During this experiment, HE staining from diabetic kidney reveals the notable mesangial matrix expansion, glomerular hypertrophy, which indicates the existence of abnormal glomerular mesangial cells proliferation. Meantime, a remarkable glycogen deposition in the glomerular region of diabetic kidney is detected by PAS staining demonstrates the presence of HG. Besides, immunohistochemistry also shows an obvious positive expression of GLUT4 in the tubulointerstitial and glomerular region. Based on the above research results, we hold that persistent hyperglycemia may induce additional glucose uptake mediated by GLUT4, which could contribute to a state of glucotoxicity for the GMCs after a hyperglycemic episode (Wang AJ et al., 2019; Arnoni CP et al., 2009). After the intervention, the renoprotective effects of BBR were assessed by histopathological and find that BBR can markedly ameliorate the notable mesangial matrix expansion and glomerular hypertrophy. The above changes in BBR treatment groups are similar to those in the metformin-positive control group. What is the mechanism by which BBR protects the kidneys against mesangial expansion?

It has been demonstrated that mesangial hypercellularity precedes an increase in the level of extracellular matrix proteins and glomerular sclerosis ( $\mathrm{Li} \mathrm{J}$ et al., 2020). The level of intracellular glucose is strongly associated with GMCs proliferation, so GMCs cultured in HG (30 mmol/L) are employed to study DN at the cellular levels (Gong W et al., 2018; Ayo SH et al., 1990). In Fig. 4, HG $(30 \mathrm{mmol} / \mathrm{L})$ significantly induces the abnormal GMCs proliferation at $24 \mathrm{~h}$ compared to that of $12 \mathrm{~h}$ and normal glucose condition ( $5.5 \mathrm{mmol} / \mathrm{L}$ ), which indicates that $\mathrm{HG}$ is an important factor for the abnormal GMCs proliferation and the induction phenomenon needs a process. Table 1 showed that BBR exerts an ideal inhibitory effect on HGinduced GMCs proliferation in the concentration range from $30 \mu \mathrm{mol} / \mathrm{L}$ to $90 \mu \mathrm{mol} / \mathrm{L}$ after adjusting for its cytotoxic by trypan blue staining. The aforementioned results revealed that the appropriate concentration of BBR (30-90 $\mu \mathrm{mol} / \mathrm{L})$ can effectively inhibit the HG-induced abnormal GMCs proliferation, leading to amelioration of the resulting diabetic kidney histopathologic changes, even kidney injury. Cellular cycle stability, a critical factor to affect cell functions, particularly growth, proliferation, and differentiation, which is not limited to the embryological state (Wang Y et al., 2018; Chang $\mathrm{J}$ et al., 2020). During the progression of glomerular disease and $\mathrm{DN}$, the function alterations such as growth and proliferation of GMCs occur throughout the whole life of individuals and are likely to 
occupy an important role (Li XQ et al., 2016). In the normal renal tissue, the mass of GMCs is dynamically regulated to maintain glomerular function and its small-scale expansion is regarded as a kind of compensatory mechanism for function fluctuation (Sugama $\mathrm{J}$ et al., 2021). However, the abnormal proliferation occurs in DN heralds the unnatural change and distribution in the cell cycle (Nangaku M et al., 2020). Therefore, experiments are performed to investigate the effect of HG on the abnormal GMCs proliferation and to discover its effect characteristic on cell cycle re-distribution. Based on the results of this study, we find that GMCs cell cycle changed un-distinctively before the stimulus for $20 \mathrm{~h}$. After exposure of GMCs to HG for $20 \mathrm{~h}$, the addition of glucose stimulated could re-distribute the $\mathrm{G} 1$ and $\mathrm{S}$ phase. The population of cells entering in $S$ phase is substantially increased by $H G$ and a decreased in the percentage of resting cells in G1 phase, while little changes of mitotic cells in G2 phase occurs. We predict that this may be in preparation for the abnormal GMCs proliferation and the correctness of analysis is verified by the EdU experiment. This indicates that there was an early HG-induced glucose influx into the cells in response to extracellular HG leading to the activation of intracellular mechanisms that mediate the subsequent pathological responses. Therefore, preventing glucose entry into cells at the early stage of the cell cycle is required for normal cell division. BBR treatment can not only increase the proportion of $\mathrm{G} 1$ phase, but also reduce the proportions of $\mathrm{S}$ phase, and this tendency becomes more significant as time goes on. This supports our hypothesis that major pathological responses are generated from hyperglycemic dividing cells. Since excessive glucose was inside of cells in the HG cultures, the question is whether this intracellular glucose active the intermediate energy of abnormal GMCs proliferation during the early stage of the DN. As a consequence, these results indicate that BBR could reverse the HG-induced abnormal cell cycle re-distribution, and this action mainly focuses on the G1 phase and $\mathrm{S}$ phase rather than the G2 phase. Therefore, it may be a good proposal to interfere the G1 and $S$ phase re-distribution to ameliorate the abnormal GMCs proliferation.

According to a number of studies, GLUT4 is the only transporter responsible for facilitating glucose transport into cells in response to insulin (Thorens B et al., 2010), and is considered as a vital regulator of entire body glucose homeostasis (Chung JJ et al., 2020; Knudsen JR et al., 2020). Meantime, studies suggest that the recruitment of GLUT4 to the membrane is essential for the glucose uptake process (Thorens B et al., 2010). Decreased protein expression and translocation of GLUT4 to the cell surface in cardiomyocytes have been found in both types $\nabla$ and $\nabla$ diabetes mellitus (Essandoh $\mathrm{K}$ et al., 2020; Wende AR et al., 2020; Yuan Y et al., 2019). Additionally, research shows that AS160 knockdown was shown to result in a partial re-distribution of GLUT4 from intracellular compartments to the membrane, a concomitant increase in basal glucose uptake, and a 3-fold increase in basal GLUT4 exocytosis (Wende AR et al., 2020). Besides, a study indicates that PI3K/AKT pathway works as upstream signaling to stimulate the GLUT4 translocation to the cell surface in cardiomyocytes, thereby promoting glucose uptake (Yuan Y et al., 2019). During this procedure, GLUT4 translocation is mediated by AS160, which is activated through AKT phosphorylation (Hatakeyama $\mathrm{H}$ et al., 2019). Based on these discoveries, we propose that PI3K/AKT/AS160/GLUT4 signaling pathway plays an important role in the glucose uptake process during diabetes mellitus and its complications, like DN. In the present study, BBR plays a similar role to that of PI3K inhibitor LY294002, which indicated BBR can significantly inhibit the HG-induced PI3K- 
p85 expression. Subsequently, the elevated expression levels of p-AKT, p-AS160, and membrane-GLUT4 were significantly declined after the treatment of BBR, while their total protein expression forms change undistinguishable. At the same time, we found that the changing trend of mRNA levels is consistent with their protein alteration. These original observations provide an important piece of the puzzle, recapitulating the mechanisms by which GLUT4, an early marker of DN, regulates glucose uptake. Therefore, our study finds that BBR inhibits the GLUT4 translocation to the plasm and cell cycle rearrangement is related to PI3K/AKT signaling, eventually relieve the corresponding pathological changes.

\section{Conclusion}

The present study demonstrates that BBR protects renal damage in DN mice. A further vitro study shows BBR suppress the activation of PI3K/AKT/AS160/GLUT4 pathway to inhibit the abnormal GMCs proliferation by retarding cell cycle re-distribution to make it stay at G1 phase. Targeting the cell cycle redistribution of GMCs is a potential therapeutic strategy and BBR can also be considered as a promising therapeutic drug in the treatment of DN.

\section{Abbreviations}

BBR

berberine; GMCs:glomerular mesangial cells; DN:diabetic nephropathy; ESRD:end-stage renal disease; ECM:excessive extracellular matrix; HG:high glucose; GLUTs:Glucose transporters; TEM:Transmission electron microscopy.

\section{Declarations}

\section{Acknowledgements}

We thank American Journal Expert (AJE) for linguistic assistance during the preparation of this manuscript.

\section{Authors' contributions}

Wei-jian Ni and Hong Zhou designed research; Xi-mei Guan performed research; Wei-jian Ni contributed new reagents or analytic tools; Xi-mei Guan and Jing Zeng analyzed data; Wei-jian Ni wrote the paper; Hong Zhou and Li-qin Tang revised the manuscript. All authors read and approved the final manuscript.

\section{Funding}

This work was supported by the National Natural Science Foundation of China (No. 81803602, 81773955), the Anhui Provincial Natural Science Foundation (No. 1708085QH207), the Fundamental 
Research Funds for the Central Universities (No. WK9110000018), and the Scientific Research Foundation of Anhui Provincial Cancer Hospital (No. 2020YJQN008).

Conflict of interest: The authors declare that they have no known competing financial interests or personal relationships that could have appeared to influence the work reported in this paper.

\section{References}

1. Ayo, S.H., et al., High glucose causes an increase in extracellular matrix proteins in cultured mesangial cells. Am J Pathol, 1990. 136(6): 1339-48.

2. Arnoni, C.P., et al., Regulation of glucose uptake in mesangial cells stimulated by high glucose: role of angiotensin II and insulin. Exp Biol Med (Maywood), 2009. 234(9): 1095-101.

3. Clark, A.J. and S.M. Parikh, Targeting Energy Pathways in Kidney Disease: The Roles of Sirtuins, AMPK, and PGC1alpha. Kidney Int, 2020.

4. Coughlan, M.T. and K. Sharma, Challenging the dogma of mitochondrial reactive oxygen species overproduction in diabetic kidney disease. Kidney Int, 2016. 90(2): 272-279.

5. Chang, J., et al., Long non-coding RNA CDKN2B-AS1 regulates high glucose-induced human mesangial cell injury via regulating the miR-15b-5p/WNT2B axis. Diabetol Metab Syndr, 2020. 12(1): 109.

6. Chung, J.J., et al., Single-Cell Transcriptome Profiling of the Kidney Glomerulus Identifies Key Cell Types and Reactions to Injury. J Am Soc Nephrol, 2020. 31(10): 2341-2354.

7. Essandoh, K., et al., Tsg101 Is Involved in the Sorting and Re-Distribution of Glucose Transporter-4 to the Sarcolemma Membrane of Cardiac Myocytes. Cells, 2020. 9(9).

8. Fu, H., et al., Diabetic kidney diseases revisited: A new perspective for a new era. Mol Metab, 2019. 30: 250-263.

9. Fruman, D.A., et al., The PI3K Pathway in Human Disease. Cell, 2017. 170(4): 605-635.

10. Gong, W., et al., CKIP-1 affects the polyubiquitination of Nrf2 and Keap1 via mediating Smurf1 to resist HG-induced renal fibrosis in GMCs and diabetic mice kidneys. Free Radic Biol Med, 2018. 115: 338-350.

11. Han, Y., et al., Reactive oxygen species promote tubular injury in diabetic nephropathy: The role of the mitochondrial ros-txnip-nlrp3 biological axis. Redox Biol, 2018. 16: 32-46.

12. Hao, P., et al., Traditional Chinese Medicine for Cardiovascular Disease: Evidence and Potential Mechanisms. J Am Coll Cardiol, 2017. 69(24): 2952-2966.

13. Hatakeyama, H., et al., Cooperative actions of Tbc1d1 and AS160/Tbc1d4 in GLUT4-trafficking activities. J Biol Chem, 2019. 294(4): 1161-1172.

14. Ji, T.T., et al., Loss of IncRNA MIAT ameliorates proliferation and fibrosis of diabetic nephropathy through reducing E2F3 expression. J Cell Mol Med, 2020. 
15. Kanwar, Y.S., et al., Diabetic nephropathy: mechanisms of renal disease progression. Exp Biol Med (Maywood), 2008. 233(1): 4-11.

16. Kushwaha, K., S. Sharma and J. Gupta, Metabolic memory and diabetic nephropathy: Beneficial effects of natural epigenetic modifiers. Biochimie, 2020. 170: 140-151.

17. Knudsen, J.R., et al., Prior exercise in humans redistributes intramuscular GLUT4 and enhances insulin-stimulated sarcolemmal and endosomal GLUT4 translocation. Mol Metab, 2020. 39: 100998.

18. Lv, Y., et al., Antidiabetic effects of a lipophilic extract obtained from flowers of Wisteria sinensis by activating Akt/GLUT4 and Akt/GSK3beta. Food Nutr Res, 2020. 64.

19. Lu, C.C., et al., Insulin induction instigates cell proliferation and metastasis in human colorectal cancer cells. Int J Oncol, 2017. 50(2): 736-744.

20. Larance, M., et al., Characterization of the role of the Rab GTPase-activating protein AS160 in insulinregulated GLUT4 trafficking. J Biol Chem, 2005. 280(45): 37803-13.

21. Li, J., et al., GdCl3 attenuates the glomerular sclerosis of streptozotocin (STZ) induced diabetic rats via inhibiting TGF-beta/Smads signal pathway. J Pharmacol Sci, 2020. 142(2): 41-49.

22. Li, X.Q., et al., Corosolic acid inhibits the proliferation of glomerular mesangial cells and protects against diabetic renal damage. Sci Rep, 2016. 6: 26854.

23. Molinaro, A., et al., Insulin-Driven PI3K-AKT Signaling in the Hepatocyte Is Mediated by Redundant PI3Kalpha and PI3Kbeta Activities and Is Promoted by RAS. Cell Metab, 2019. 29(6): 1400-1409.e5.

24. Ni, W.J., et al., Renoprotective effect of berberine via regulating the PGE2 -EP1-Galphaq-Ca(2+) signalling pathway in glomerular mesangial cells of diabetic rats. J Cell Mol Med, 2016. 20(8): 1491502 .

25. Nangaku, M., Schlondorff and Lee revealed crosstalk between glomerular cells and a role of BAMBI in diabetic kidney disease. Kidney Int, 2020. 98(3): 539-541.

26. Perkovic, V., et al., Canagliflozin and Renal Outcomes in Type 2 Diabetes and Nephropathy. N Engl J Med, 2019. 380(24): 2295-2306.

27. Perkins, B.A., et al., Risk Factors for Kidney Disease in Type 1 Diabetes. Diabetes Care, 2019. 42(5): 883-890.

28. Qi, M.Y., et al., Protective effect of ferulic acid on STZ-induced diabetic nephropathy in rats. Food Funct, 2020. 11(4): 3706-3718.

29. Qi, C., et al., Classification and Differential Diagnosis of Diabetic Nephropathy. J Diabetes Res, 2017. 2017: 8637138.

30. Qiu, Y.Y., L.Q. Tang and W. Wei, Berberine exerts renoprotective effects by regulating the AGEs-RAGE signaling pathway in mesangial cells during diabetic nephropathy. Mol Cell Endocrinol, 2017. 443: 89-105.

31. Sagoo, M.K. and L. Gnudi, Diabetic nephropathy: Is there a role for oxidative stress? Free Radic Biol Med, 2018. 116: 50-63. 
32. Shinjyo, N., et al., Berberine for prevention of dementia associated with diabetes and its comorbidities: A systematic review. J Integr Med, 2020. 18(2): 125-151.

33. Sugama, J., et al., Enteropeptidase inhibition improves kidney function in a rat model of diabetic kidney disease. Diabetes Obes Metab, 2021. 23(1): 86-96.

34. Tziomalos, K. and V.G. Athyros, Diabetic Nephropathy: New Risk Factors and Improvements in Diagnosis. Rev Diabet Stud, 2015. 12(1-2): 110-8.

35. Thorens, B. and M. Mueckler, Glucose transporters in the 21st Century. Am J Physiol Endocrinol Metab, 2010. 298(2): E141-5.

36. Tang, D., et al., Rapid Analysis and Guided Isolation of Astragalus Isoflavonoids by UHPLC-DADMS(n) and Their Cellular Antioxidant Defense on High-Glucose-Induced Mesangial Cell Dysfunction. J Agric Food Chem, 2018. 66(5): 1105-1113.

37. Tervaert, T.W., et al., Pathologic classification of diabetic nephropathy. J Am Soc Nephrol, 2010. 21(4): 556-63.

38. Tsai, Y.C., et al., High Glucose Induces Mesangial Cell Apoptosis through miR-15b-5p and Promotes Diabetic Nephropathy by Extracellular Vesicle Delivery. Mol Ther, 2020. 28(3): 963-974.

39. Turgut, F. and W.K. Bolton, Potential new therapeutic agents for diabetic kidney disease. Am J Kidney Dis, 2010. 55(5): 928-40.

40. Vallon, V. and S.C. Thomson, Targeting renal glucose reabsorption to treat hyperglycaemia: the pleiotropic effects of SGLT2 inhibition. Diabetologia, 2017. 60(2): 215-225.

41. Wang, A.J., et al., Heparin affects cytosolic glucose responses of hyperglycemic dividing mesangial cells. J Biol Chem, 2019. 294(16): 6591-6597.

42. Wu, Z., et al., Macrovascular Protecting Effects of Berberine through Anti-inflammation and Intervention of BKCa in Type 2 Diabetes Mellitus Rats. Endocr Metab Immune Disord Drug Targets, 2020.

43. Wang, Y., C.J. Zhou and Y. Liu, Wnt Signaling in Kidney Development and Disease. Prog Mol Biol Transl Sci, 2018. 153: 181-207.

44. Wende, A.R., et al., Maintaining Myocardial Glucose Utilization in Diabetic Cardiomyopathy Accelerates Mitochondrial Dysfunction. Diabetes, 2020. 69(10): 2094-2111.

45. Xia, X., et al., Jiaogulan tea (Gpostemma pentaphyllum) potentiates the antidiabetic effect of white tea via the AMPK and PI3K pathways in C57BL/6 mice. Food Funct, 2020. 11(5): 4339-4355.

46. Xie, R., et al., The protective effect of betulinic acid (BA) diabetic nephropathy on streptozotocin (STZ)-induced diabetic rats. Food Funct, 2017. 8(1): 299-306.

47. Young, L.H., D.L. Coven and R.R. Russell, Cellular and molecular regulation of cardiac glucose transport. J Nucl Cardiol, 2000. 7(3): 267-76.

48. Yuan, Y., et al., PEDF increases GLUT4-mediated glucose uptake in rat ischemic myocardium via PI3K/AKT pathway in a PEDFR-dependent manner. Int J Cardiol, 2019. 283: 136-143. 
49. Zhang, H., et al., Activation of PI3K/Akt pathway limits JNK-mediated apoptosis during EV71 infection. Virus Res, 2014. 192: 74-84.

50. Zhang, J., et al., Paeoniflorin influences breast cancer cell proliferation and invasion via inhibition of the Notch1 signaling pathway. Mol Med Rep, 2018. 17(1): 1321-1325.

\section{Tables}

Table 1 Results of trypan blue staining of different times

Table 1A. Cell survival at 24 hours

\begin{tabular}{|lll|}
\hline & $120 \mu \mathrm{M}$ & $150 \mu \mathrm{M}$ \\
\hline Ration of live cells (\%) & $78.0 \pm 3.0$ & $67.0 \pm 1.5$ \\
\hline Ration of dead cells (\%) & $22.0 \pm 3.0$ & $31.0 \pm 5.0$ \\
\hline
\end{tabular}

Table 1B. Cell survival at 36 hours

\begin{tabular}{|llll|}
\hline & $90 \mu \mathrm{M}$ & $120 \mu \mathrm{M}$ & $150 \mu \mathrm{M}$ \\
\hline Ration of live cells (\%) & $75.0 \pm 4.0$ & $67.0 \pm 1.5$ & $60.0 \pm 3.0$ \\
\hline Ration of dead cells (\%) & $25.0 \pm 4.0$ & $34.0 \pm 1.5$ & $40.0 \pm 3.0$ \\
\hline
\end{tabular}

Table 1C. Cell survival at 48 hours

\begin{tabular}{|llll|}
\hline & $90 \mu \mathrm{M}$ & $120 \mu \mathrm{M}$ & $150 \mu \mathrm{M}$ \\
\hline Ration of live cells (\%) & $36.0 \pm 5.5$ & $14.0 \pm 0.5$ & $7.0 \pm 1.0$ \\
\hline Ration of dead cells (\%) & $65.0 \pm 5.5$ & $87.0 \pm 0.5$ & $93.0 \pm 1.0$ \\
\hline
\end{tabular}

Table 2 qRT-PCR primer sequences

\begin{tabular}{|lll|}
\hline Gene Name & Forward primer & Reverse primer \\
\hline GAPDH & AGGTCGGTGTGAACGGATTTG & GGGGTCGTTGATGGCAACA \\
\hline PI3K-p85 & ACACCACGGTTTGGACTATGG & GGCTACAGTAGTGGGCTTGG \\
\hline AKT & ATGAACGACGTAGCCATTGTG & TTGTAGCCAATAAAGGTGCCAT \\
\hline GLUT4 & ACACTGGTCCTAGCTGTATTCT & CCAGCCACGTTGCATTGTA \\
\hline AS160 & GAGTCGCCTAGCTGCATTCAG & CCACGTACCATAGCCGGAA \\
\hline
\end{tabular}




\section{Figures}

A
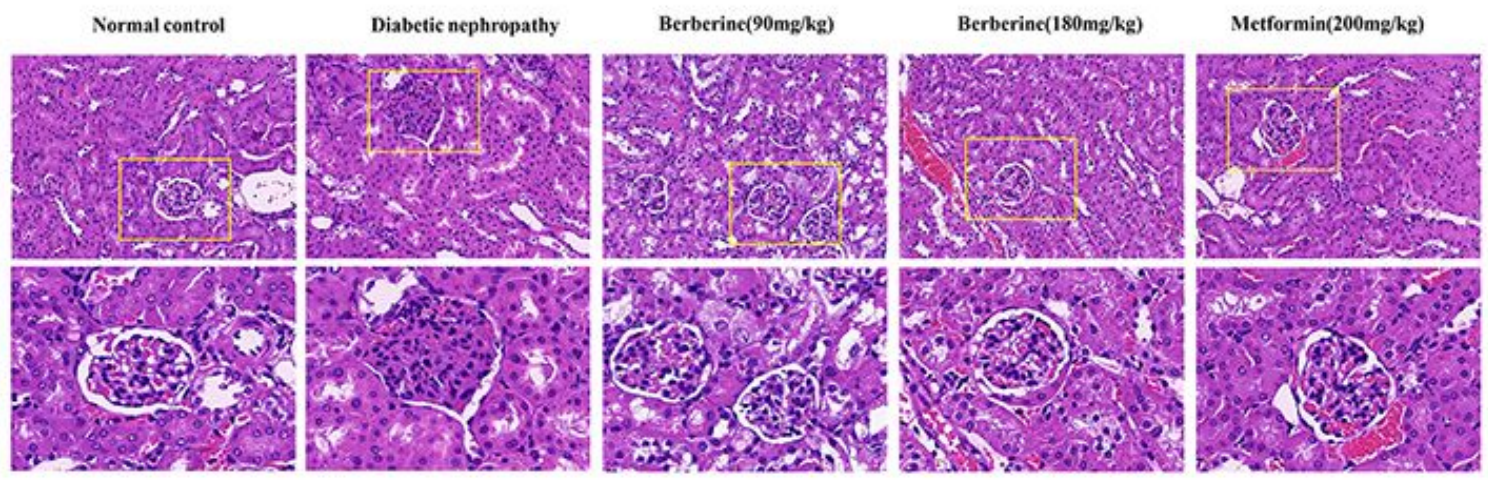

B
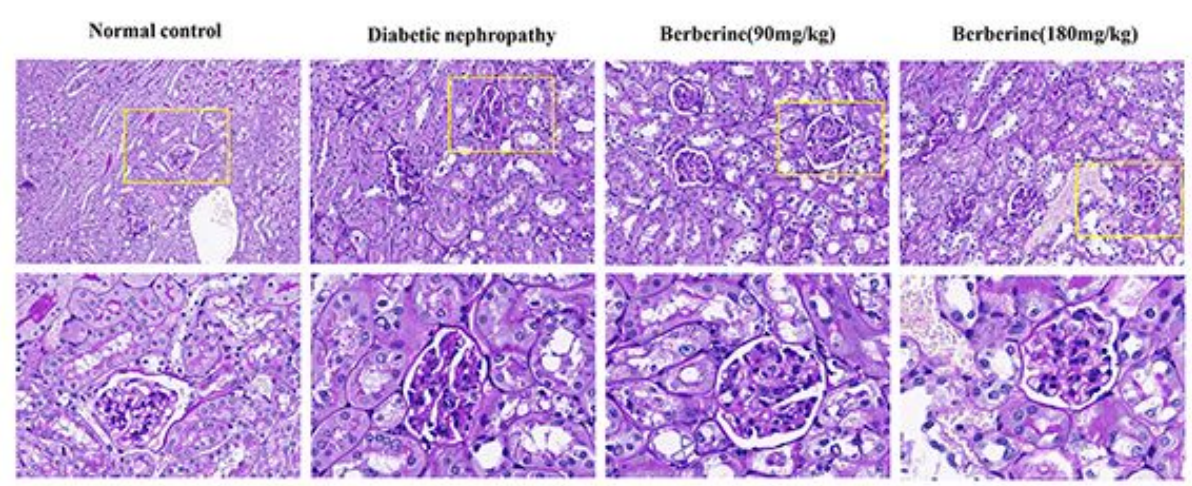

Metformin(200mg/kg)
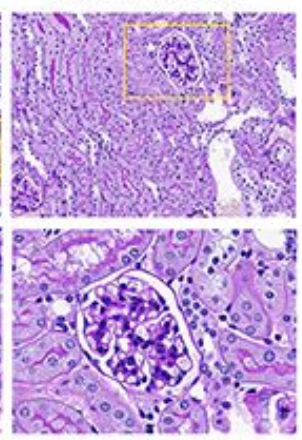

C
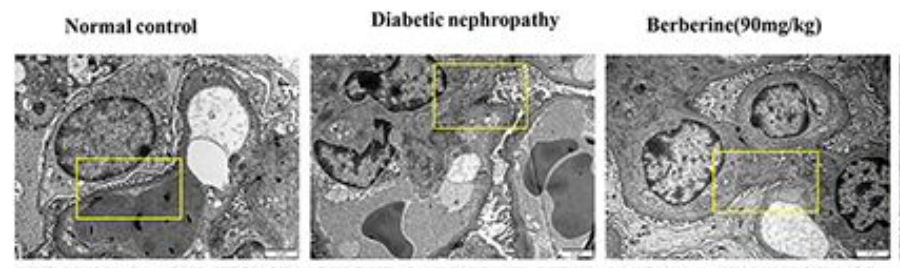

Berberine(180mg/kg)

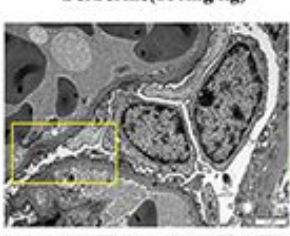

Metformin(200mg/kg)
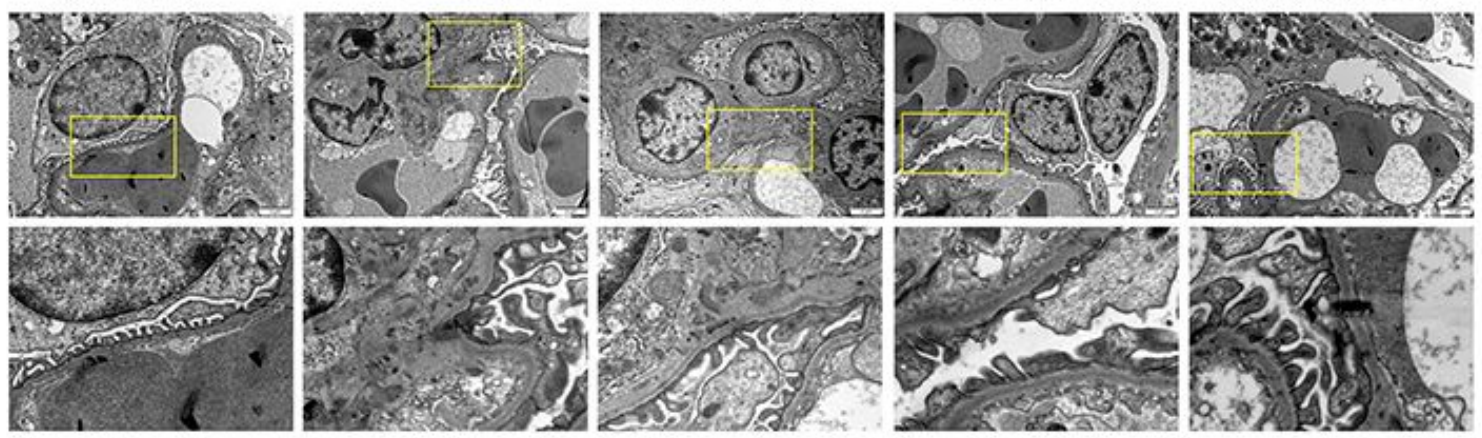

\section{Figure 1}

Effect of BBR on DN mice kidney tissue. ( $\bar{x} \pm s, n=6)$. The STZ-induced diabetic mice were intragastrically administered with BBR $(90,180 \mathrm{mg} / \mathrm{kg})$ and Metformin $(200 \mathrm{mg} / \mathrm{kg})$ once daily for 12 weeks. The pathological analysis of glomerular was performed using Hematoxylin-eosin staining (HE) and Periodic acid-Schiff staining (PAS). The glomerular ultrastructure of DN mice was observed by transmission electron microscopy (TEM). (A) HE staining of mice kidney tissues; (B) PAS staining of mice kidney 
tissues. (C) TEM of the basement membrane thickening of kidney tissues. The images were taken at $40 \mathrm{X}$ magnification. metformin was taken as a positive control. The data represent the mean $\pm S D$. $n=6$.

A

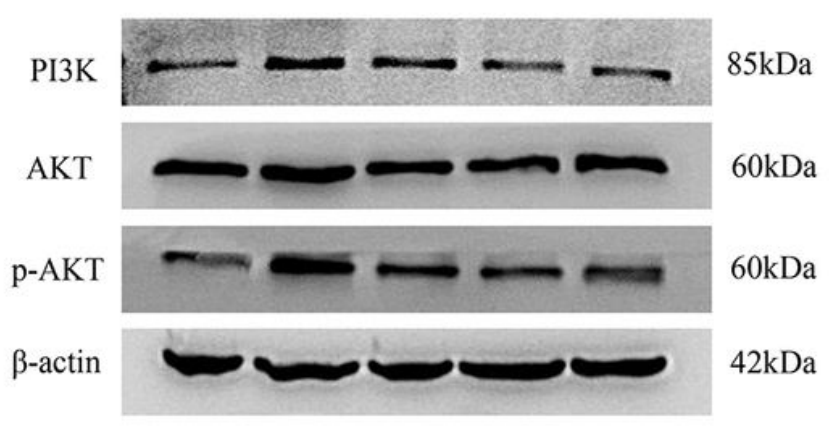

$\mathrm{C}$

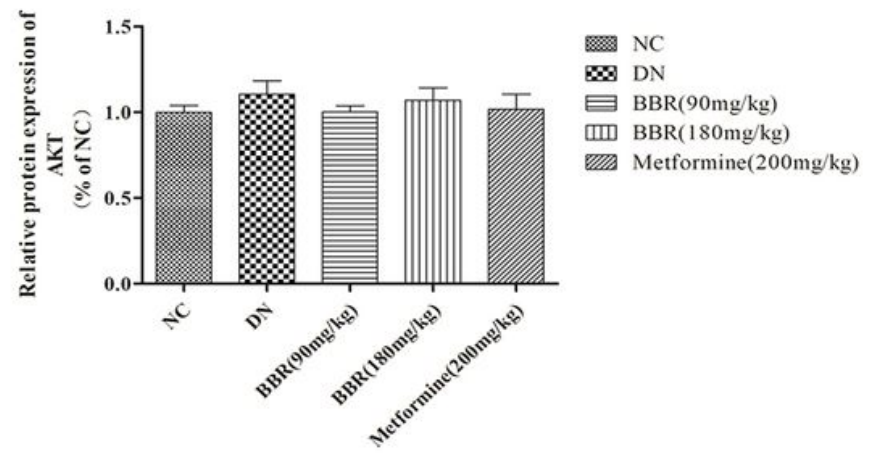

B

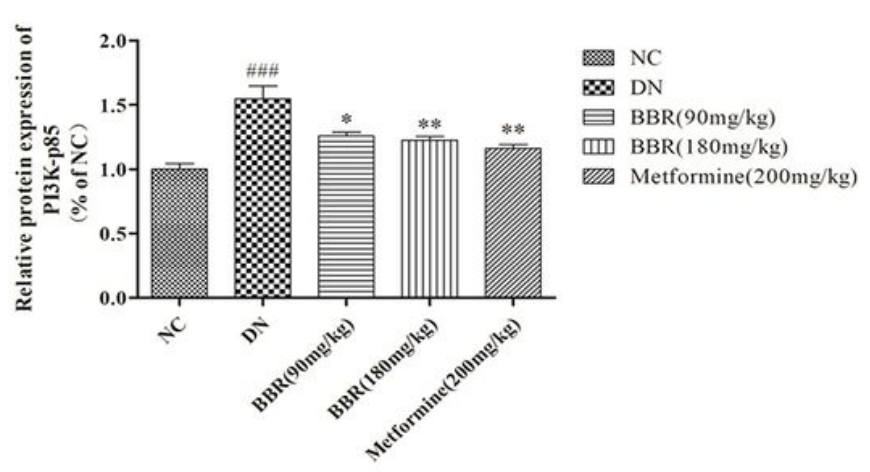

$\mathrm{D}$

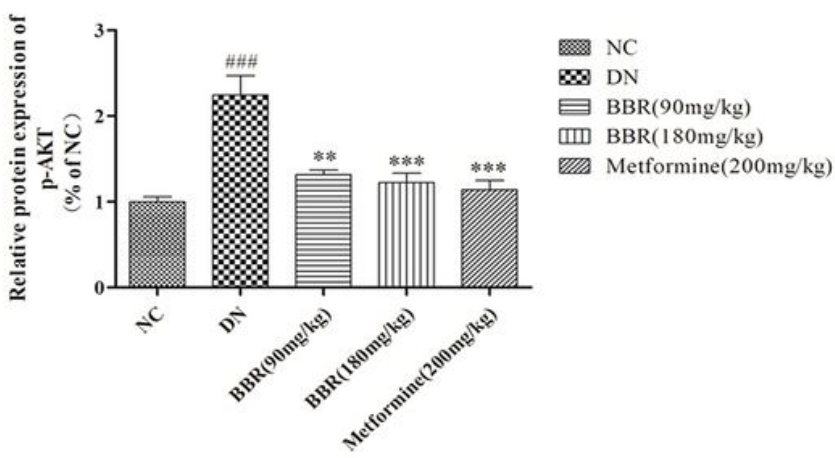

Figure 2

Effect of BBR on PI3K/AKT signaling pathway in DN mice kidneys. $(\overline{\mathrm{x}} \pm \mathrm{s}, \mathrm{n}=3)$. Left lane showing a representative Western blot and the right lane showing the densitometry measurements normalized to $\beta$ actin. The data represent the mean \pm SD. $n=3$. \#\#\#P<0.001 vs NC group, ${ }^{*} P<0.05,{ }^{* *} P<0.01,{ }^{* \star *} P<0.001$ vs DN group. 
A

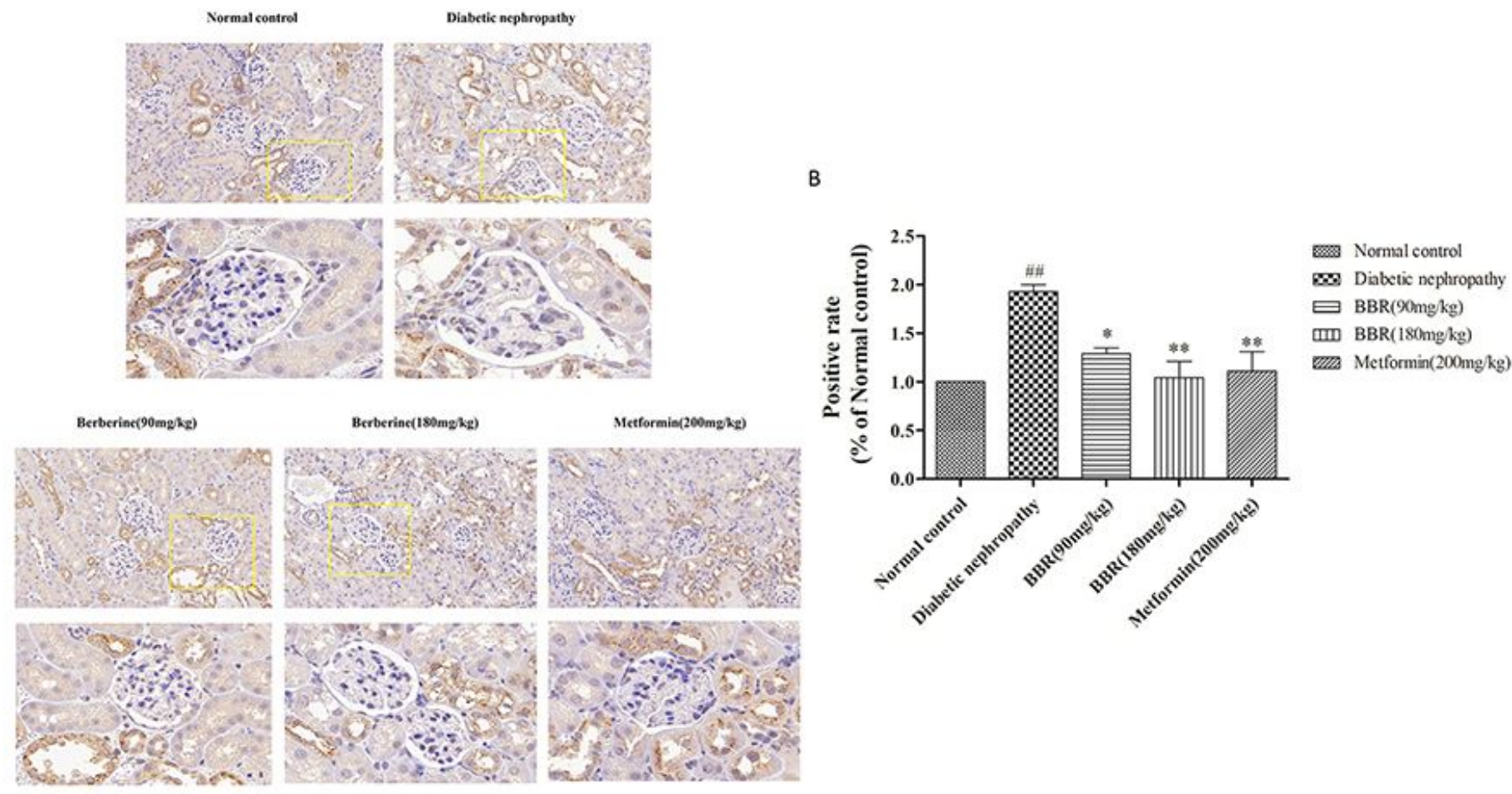

\section{Figure 3}

Effect of BBR on GLUT4 expression of mice kidneys. ( $\overline{\mathrm{x}} \pm \mathrm{s}, \mathrm{n}=6)$. Mice were subjected to diabetic nephropathy, and orally administered with BBR $(90,180 \mathrm{mg} / \mathrm{kg})$ or Metformin $(200 \mathrm{mg} / \mathrm{kg})$. Mice were sacrificed, and kidneys were isolated. The relative expression of GLUT4 in kidney tissues by immunohistochemistry. The data represent the mean $\pm S D$. $n=6$. \#\#P<0.01 vs NC group, ${ }^{*}<<0.05$, $\star \star P<0.01$ vs $D N$ group.

A

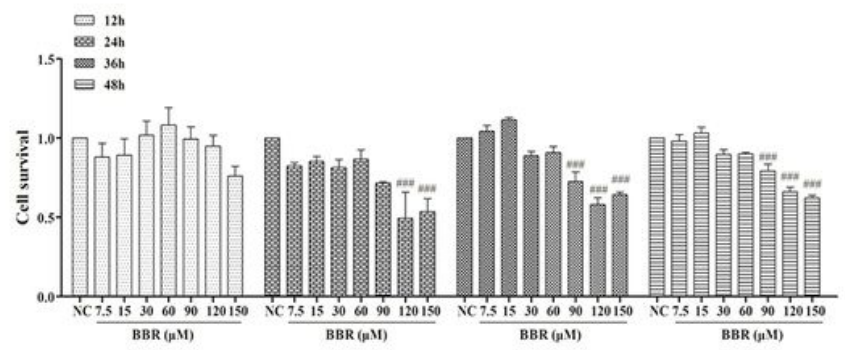

B

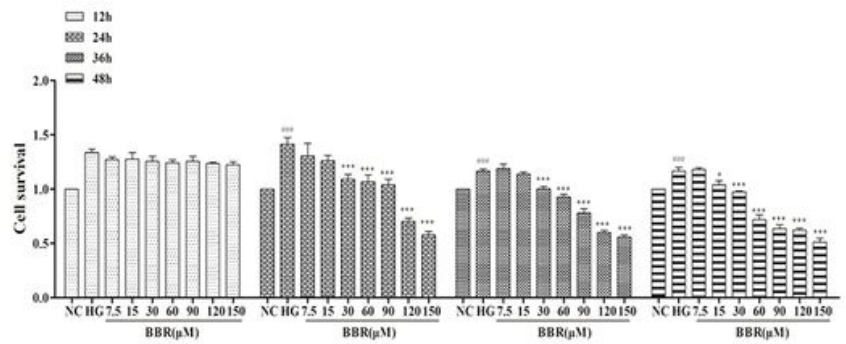

\section{Figure 4}


Effect of BBR on GMCs proliferation at different times. $(\overline{\mathrm{x}} \pm \mathrm{s}, \mathrm{n}=3)$. The cell survival is detected by MTT. (A) Effect of different concentrations of BBR on the activity of normal GMCs at different times; (B) Effect of different concentrations of BBR on GMCs proliferation in $\mathrm{HG}$ at different times. The data represent the mean \pm SD. $n=6$. \#\#\#P<0.001 vs NC, ${ }^{*} P<0.05$, ${ }^{* \star *} P<0.001$ vs HG; NC: normal glucose; HG: high glucose.

A

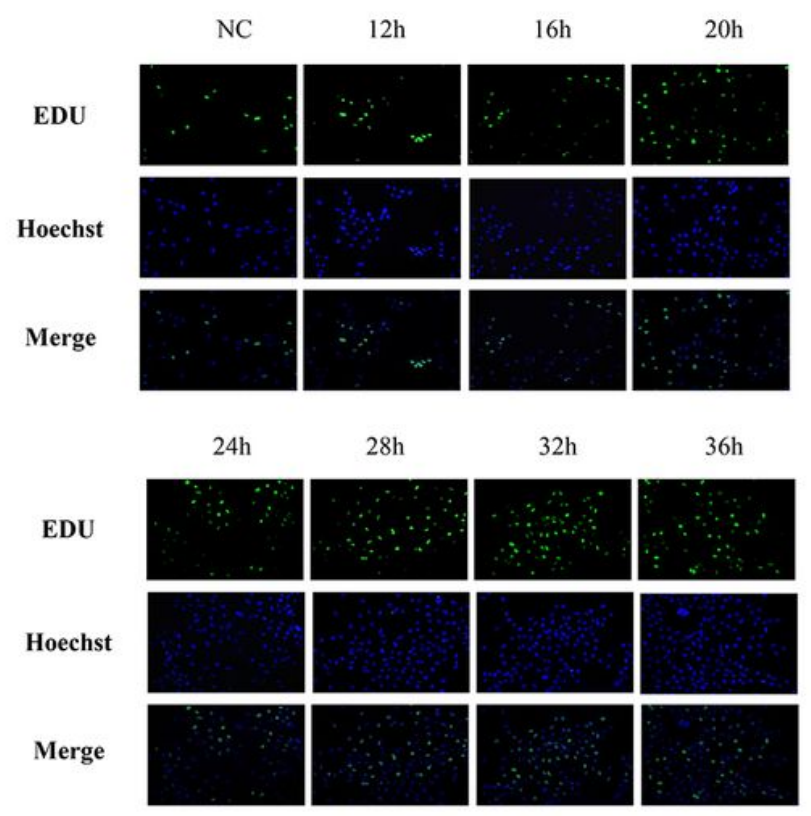

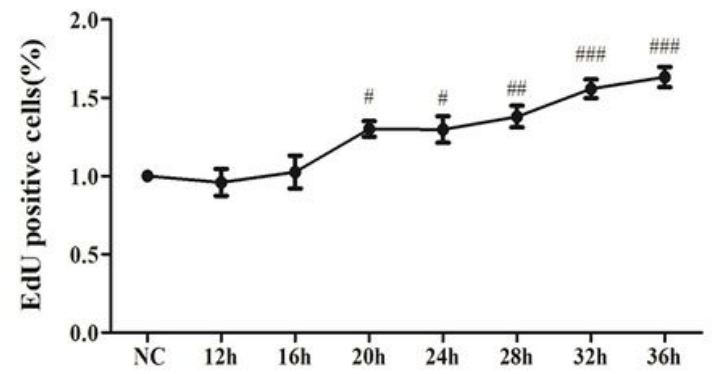

\section{Figure 5}

Effect of HG on GMCs proliferation at different times. $(\bar{x} \pm s, n=3)$. To determine the time-dependent effect, GMCs were cultured in high glucose for 0 h, 12 h, 16 h, 20 h, 24 h, 28 h, 32 h, 36 h. EdU staining to observe the GMCs proliferation. (A) Representative EdU staining image of GMCs treated with high glucose were shown. (B) The quantification results of EdU-positive cells (\%). Experiments were repeated for at least three times. \#\#\#P<0.001 vs NC group, ${ }^{*} \mathrm{P}<0.05$, ${ }^{\star *} * \mathrm{P}<0.001$ vs $\mathrm{HG}$ group; $\# \mathrm{P}<0.05 \rrbracket \# \# \mathrm{P}<0.01$, $\# \# \# P<0.001$ vs NC group. NC: normal glucose; HG: high glucose. 

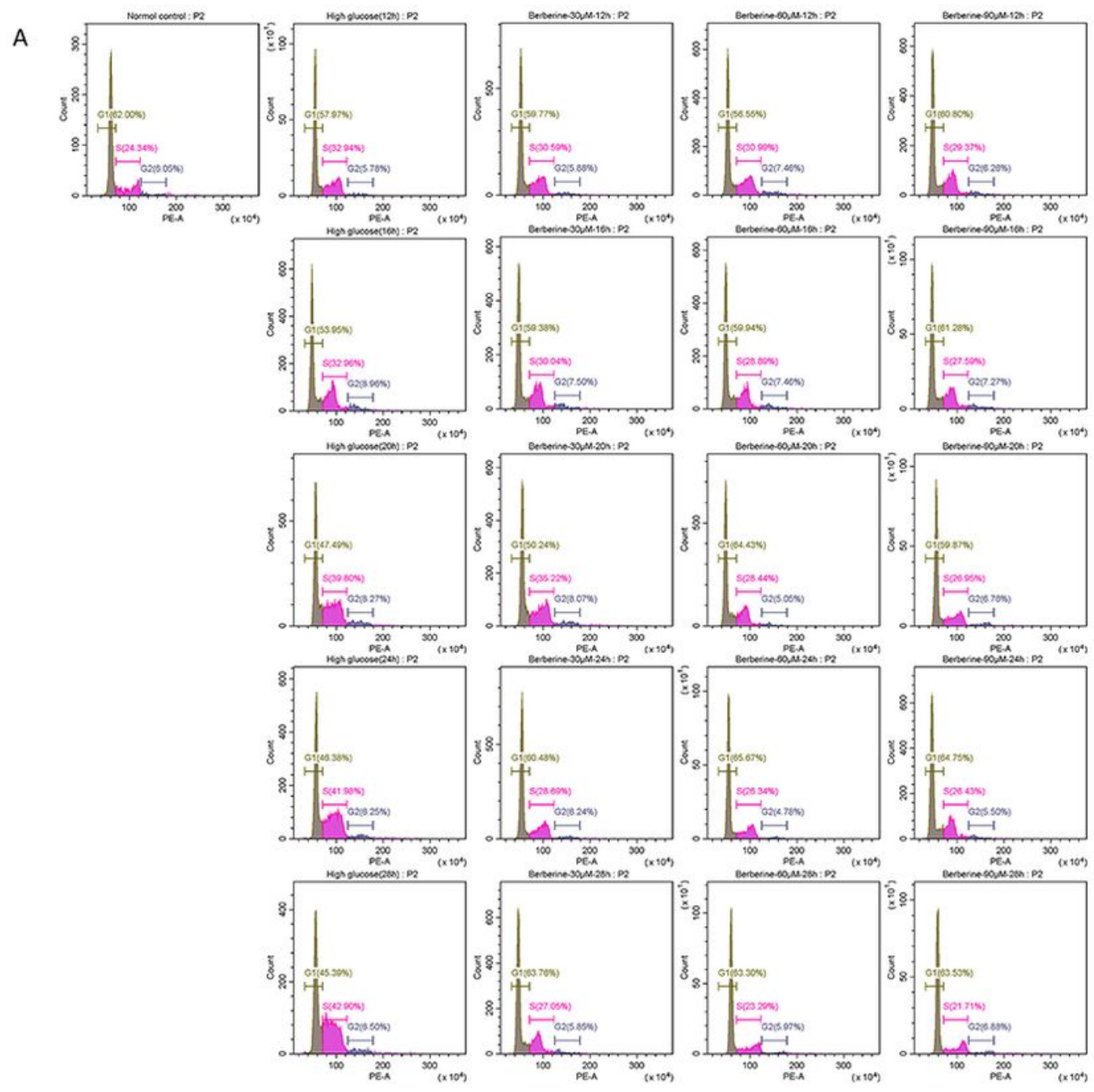

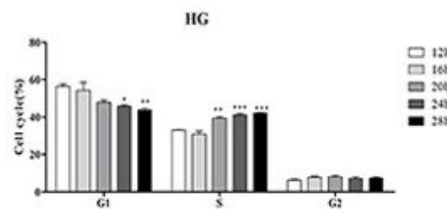

$E$

$20 \mathrm{~h}$

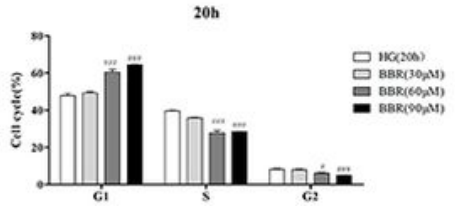

C

$12 \mathrm{~b}$

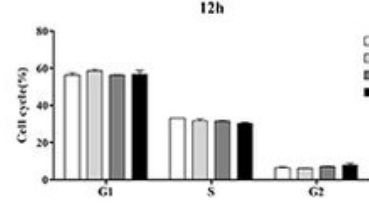

$\mathrm{F}$

2th

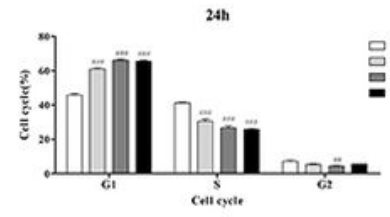

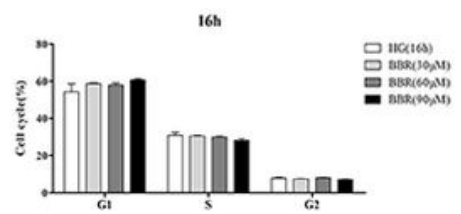

G

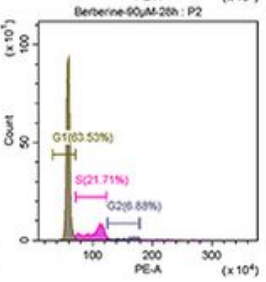

$28 \mathrm{~h}$

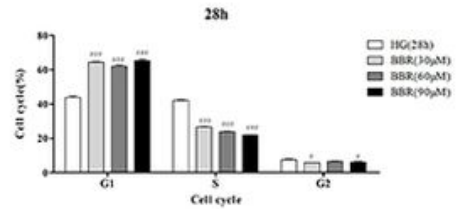

Figure 6

Flow cytometry analyze the HG-induced GMCs cell cycle re-distribution at different times. ( $\bar{x} \pm s, n=3)$. (A) The cell cycle of HG-induced GMCs after different concertation of BBR treatment at different times; (B) Effect of high glucose in GMCs cell cycle. (C-G) Effect of BBR on HG-induced GMCs cell cycle redistribution at different times. Results shown are representative of at least three individual experiments.

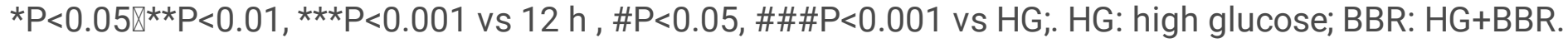


A

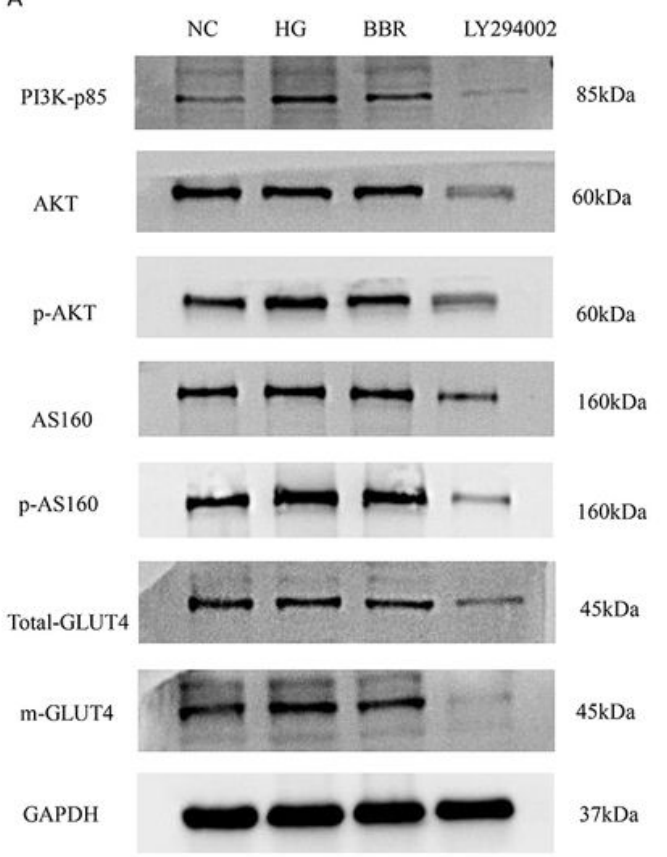

B

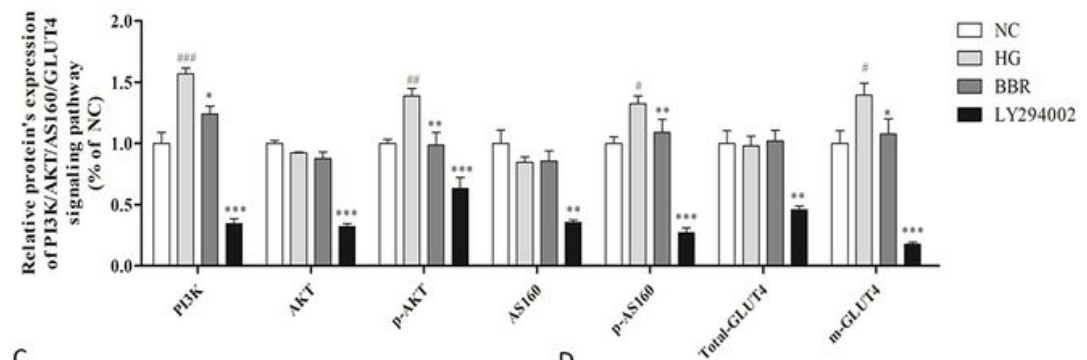

c

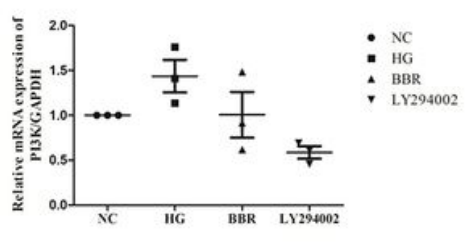

$E$
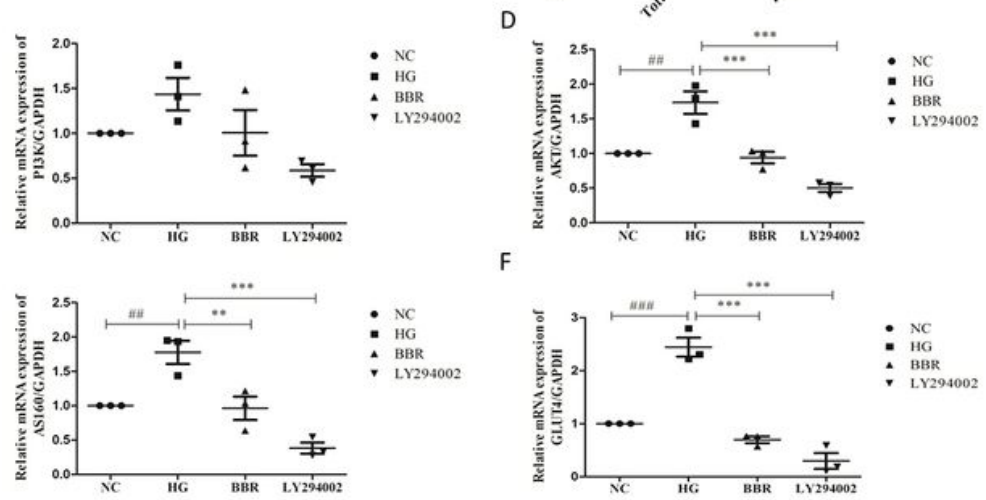

ᄃ

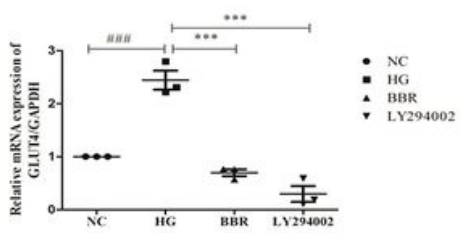

\section{Figure 7}

Effect of BBR on regulating the relative expressions in PI3K/AKT /AS160/GLUT4 signaling pathway. ( $\overline{\mathrm{x}} \pm$ $s, n=3)$. Protein was extracted from cultured GMCs. GAPDH was used as internal controls. Intensity of PI3K, AKT, p-AKT, AS160, p-AS160, Total-GLUT4 and m-GLUT4 was quantified, normalized to internal control and expressed as mean \pm SD.(A-B) The levels of PI3K, AKT, p-AKT, AS160, p-AS160, Total-GLUT4 and m-GLUT4 were detected in each group. (C-F) RT-qPCR analyses were performed to detect the mRNA expression of PI3K, AKT, AS160 and GLUT4 in each group. Relative expression of PI3K, AKT, AS160 and GLUT4. Experiments were repeated for at least three times. \#\#P<0.01, \#\#\#P<0.001 vs NC group;

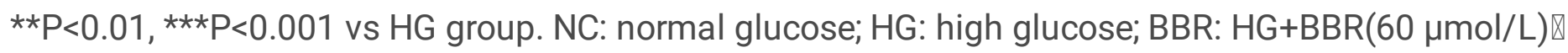
LY294002 (HG+LY294002). 
A

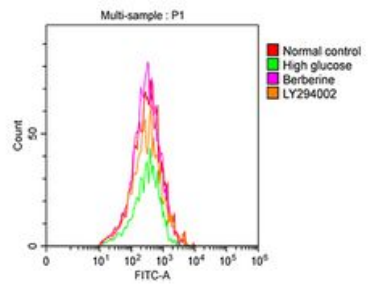

C
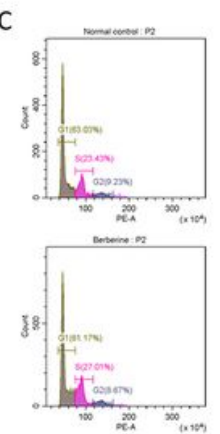

B

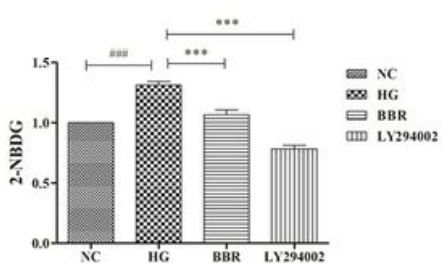

D
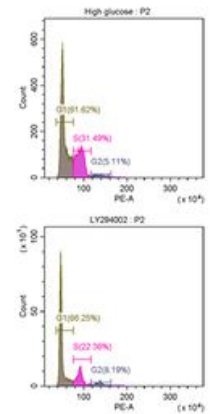

E

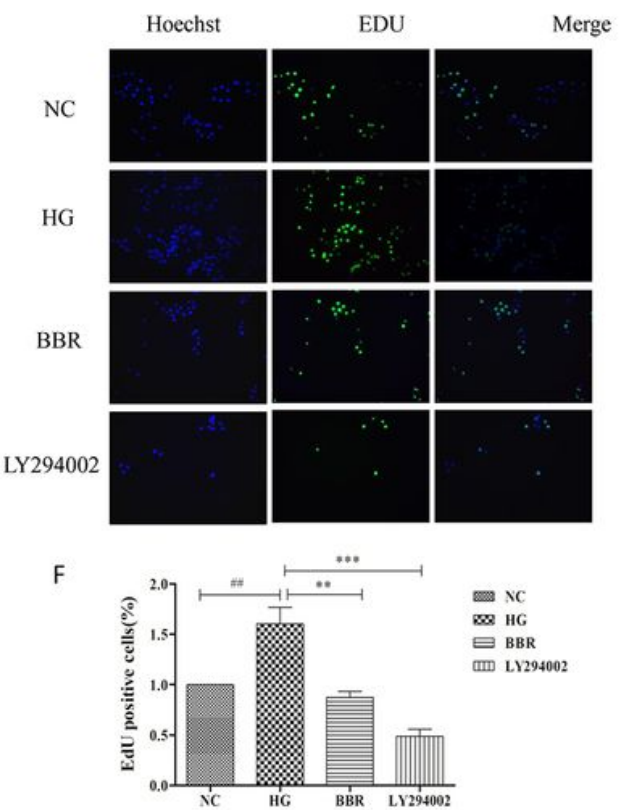

\section{Figure 8}

Effect of BBR on high glucose-induced GMCs glucose uptake. (A) Representative images of 2-NBDG. (B) Flow cytometry was used to detect glucose uptake of GMCs in high glucose for $20 \mathrm{~h}$. (C) The change of HG-induced GMCs cell cycle after BBR treatment. (D) The percentage of PI-stained cells at the G1, S, and G2 phases. (E) Representative EdU results observed using a light microscope. (F) The quantification results of EdU-positive cells. Data are expressed as means \pm SD. of three independent experiments. \#\#P<0.01, \#\#\#P<0.001 vs NC group; ${ }^{\star *} \mathrm{P}<0.01,{ }^{\star} * \star \mathrm{P}<0.001$ vs HG group. NC: normal glucose; HG: high glucose; BBR: HG+BBR (60 mol/L); LY294002 (HG+LY294002). 